Unfallchirurg 2011 · 114:966-967

DOI 10.1007/s00113-011-2031-9

Online publiziert: 4. November 2011

(c) Springer-Verlag 2011

\author{
L. Ney ${ }^{1,2} \cdot$ W. Mutschler $^{1}$ \\ ${ }^{1}$ Chirurgische Intensivmedizin, Klinik für Anaesthesiologie und \\ Chirurgische Klinik - Innenstadt, Klinikum der Universität München \\ ${ }^{2}$ Chirurgische Klinik und Poliklinik, Klinikum der LMU München
}

\title{
Ernährung nach Trauma
}

Bei der Versorgung polytraumatisierter Patienten fokussiert sich spätestens nach der Initialphase der unfallchirurgische Blick v. a. auf den operativen Versorgungsbedarf. In eine qualifizierte Indikationsstellung müssen dabei immer vorbestehende Begleiterkrankungen und aktuelle Organfunktionsstörung mit einfließen, da diese Faktoren die Prognose wesentlich mitbestimmen. Einer dieser Einflussfaktoren ist der Ernährungszustand des Patienten.

Welche Patienten auf der Intensivstation haben die bessere Prognose Patienten mit Normalgewicht oder adipöse Patienten? Die sicher für viele von uns intuitiv naheliegende Antwort „Patienten mit Normalgewicht" trifft nicht generell zu: Es ist mittlerweile gut belegt, dass adipöse Patienten mit einem „Body Mass Index“ (BMI) um $30 \mathrm{~kg} / \mathrm{m}^{2}$ im gemischten intensivmedizinischen Kollektiv die bessere Prognose haben - und mangelernährte Patienten $\left(\mathrm{BMI}<20 \mathrm{~kg} / \mathrm{m}^{2}\right)$ die schlechteste [1].

In dieser Ausgabe von Der Unfallchirurg wollen wir uns mit der praktischen Bedeutung von Ernährungszustand und Ernährungstherapie für die Akutversorgung traumatologischer Patienten beschäftigen. In großen outcomeorientierten Studien zu diesen Themen sind Traumapatienten häufig unterrepräsentiert. Daher lohnt eine genauere Analyse der wissenschaftlichen Erkenntnisse und Empfehlungen für dieses Patientenkollektiv.

Richtige und falsche Ernährung, in den Industriestaaten v. a. die chronische Hyperalimentation, hat einen enormen Einfluss auf physiologische Funktionen. Als Risikofaktor vor allem kardiovaskulärer Erkrankungen trägt Fehlernährung zu
Akutsituationen mit hohem Letalitätsrisiko bei. Daher wurde die oben dargestellte, überraschende Beobachtung der Adipositas als Prädiktor für ein günstiges Outcome als Adipositasparadoxon bezeichnet.

Hoffmann u. Rueger zeigen, dass das Adipositasparadoxon nicht bei polytraumatisierten Patienten gilt. Hier sind sowohl Unter- wie auch Übergewicht mit einer biphasischen auftretenden erhöhten Mortalität assoziiert. Während untergewichtige Patienten ein hohes initiales Letalitätsrisiko tragen, entwickeln adipöse Patienten gehäuft schwere Spätkomplikationen, namentlich Infektionen und Multiorganversagen.

\section{$>$ Bedeutung von Ernährungszustand und -therapie für die Akutversorgung traumatologischer Patienten}

Ziel der Ernährungstherapie in der Intensivmedizin ist es, den Darm nicht als "Motor des Multiorganversagens“ laufen zu lassen. Vielmehr muss möglichst bald nach einem Trauma der "Vorwärtsgang“ hin zur Sicherstellung der Darmintegrität einerseits und heraus aus der generellen Stoffwechselkatabolie andererseits eingelegt werden. Ney u. Annecke stellen die dazu vorliegenden Empfehlungen vor und diskutieren sie in Bezug zur Evidenzlage und zu ihrer praktischen Umsetzung. Eine frühe enterale Ernährung sollte der bevorzugte Weg sein. Ist dieser Weg aus praktischen Gründen verbaut, sollte eine parenterale Ernährung als temporäre Umleitung genutzt werden: Das Ziel ist eine energetisch volle Ernährung und, aus pathophysiologischer Sicht noch wichtiger, die frühe und volle Deckung des Eiweißbedarfs.

Nicht nur Kaloriezahl und Proteinversorgung tragen zur positiven Wirkung der intensivmedizinischen Ernährungstherapie bei: Ernährung und die damit unmittelbar verbundene gastrointestinale Integrität haben einen zentralen Einfluss auf das Immunsystem. Felbinger et al. stellen in ihrem Beitrag viel versprechende Optionen der Immuno- oder Pharmakonutrition dar. Neben einer ganzen Reihe anderer Substanzen können Glutamin, Fischöl und Spurenelemente wie Selen die posttraumatische Inflammation beeinflussen. Dieses Potential ist unbestritten, wenngleich nicht alle Forschungsergebnisse einheitlich lauten: Während z. B. Omega-3-Fettsäuren beim ARDS als Leitlinienempfehlung gelten, wurde die groß angelegte „EDEN-Omega Study“ des renommierten ARDS-Networks zu diesem Thema aufgrund fehlender Wirkung abgebrochen [2]. Als Einzelintervention können spezielle Komponenten der Ernährungstherapie durchaus sinnvoll sein, aber nicht isoliert über Outcomeparameter wie Beatmungsdauer oder Krankenhausletalität entscheiden.

Zentrale Fragen der Ernährungstherapie sind weiterhin wissenschaftlich nicht ausreichend beantwortet [3]. Der Vielzahl fraglos wertvoller Leitlinien steht ein gewisser Mangel an ausreichend dimensionierten, prospektiven und randomisierten Studien gegenüber. Dennoch fordern die Ergebnisse der drei Artikel dieses Leitthemas eine Verbesserung der Ernährungstherapie bei Traumapatienten geradezu heraus: Wir müssen uns konsequent an die vorliegenden Leitlinien, z. B. der interdisziplinären Deutschen Gesellschaft 
für Ernährungsmedizin (DGEM) oder der „European Society for Parenteral and Enteral Nutrition" (ESPEN), halten und gleichzeitig wissenschaftlich ihre Weiterentwicklung unterstützen.

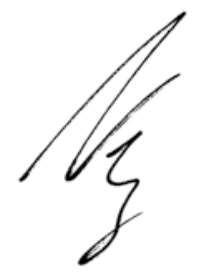

L. Ney

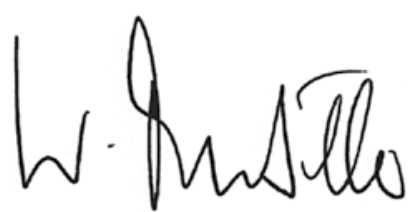

W. Mutschler

\section{Korrespondenzadressen}

\section{Dr. L. Ney}

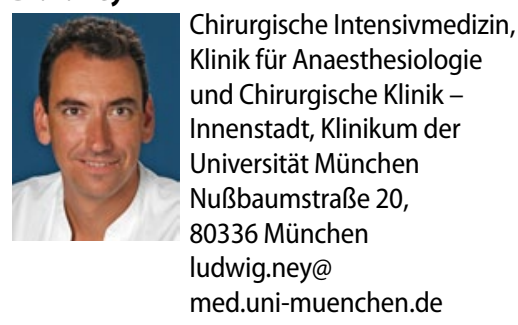

\section{Prof. Dr. W. Mutschler}

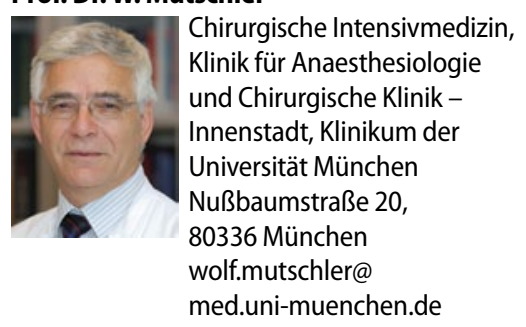

\section{Literatur}

1. Alberda C, Gramlich L, Jones N et al (2009) The relationship between nutritional intake and clinical outcomes in critically ill patients: results of an international multicenter observational study. Intensive Care Med 35:1728-1737

2. Rice TW, Wheeler AP, Thompson BT et al (2011) Enteral omrga-3 fatty acid, gamma-linolenic acid, and antioxidant supplementation in acute lung injury. JAMA 206:1574-1581

3. Ziegler TR (2011) Nutrition support in critical illness - bridging the evidence gap. N Engl J Med 365:562-564

\section{Der Orthopäde}

Die Zeitschrift „Der Orthopäde“ bietet Ihnen jeden Monat umfassende und aktuelle Beiträge zu interessanten Themenschwerpunkten aus allen Bereichen der Orthopädie. In mehreren Übersichtsartikeln wird ein Sachgebiet vertiefend dargestellt.

\section{Heft 10/2011:}

- Anatomie und Biomechanik des Patellofemoralgelenks - physiologische Gegebenheiten und Veränderungen nach Implantation einer Knie-Totalendoprothese

- Physio- und trainingstherapeutische Therapieoptionen für das instabile Patellofemoralgelenk

- Therapie der patellaren Instabilität bei Kindern und Jugendlichen

- Einteilung und Therapie der Patellafraktur

- Therapie lokaler Knorpelschäden im Patellofemoralgelenk

- Das dritte Kompartiment bei der Knieendoprothetik: Von Denervierung bis Ersatz, welche Therapie ist die richtige?

- Therapie der isolierten Retropatellararthrose, gibt es Evidenz basierte Verfahren?

- Der Patellofemorale Schmerz nach primärer Knieendoprothetik - Behandlungsalgorhitmus und Literaturüberblick

- CME: Diagnostik von malignen Knochen- und Weichteiltumoren

\section{Heft 11/2011:}

- Sprunggelenkprothese bei Varusarthrose

- Sprunggelenkprothese bei Valgusarthrose

- Sprunggelenkprothese bei Knochendefekten

- Sprunggelenkprothese bei chronischer Polyarthritis

- Mediales Schmerzsyndrom nach Sprunggelenkprothesenimplantation

- Revisionsarthroplastik des oberen Sprunggelenks

- Sprunggelenkarthrodese nach gescheiterter Endoprothesenimplantation

- CME: Fibromyalgie

\section{Der Orthopäde + Der Unfallchirurg: \\ Jetzt zum Kombipreis abonnieren und bis zu 239 Euro sparen!}

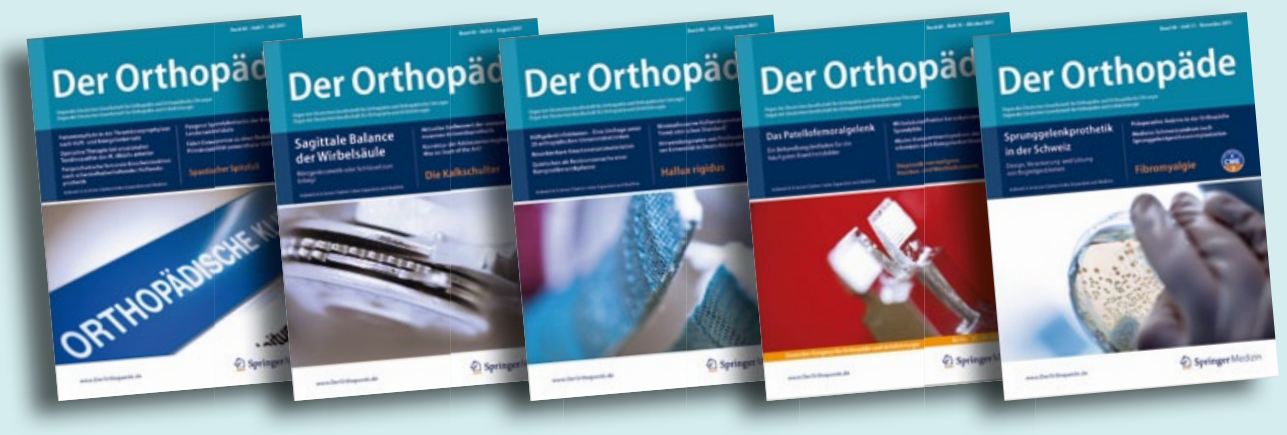

24 Hefte jährlich für 399,- EUR. Ärztinnen/Ärzte in Aus- und Weiterbildung erhalten das Kombiabo zum Vorzugspreis von 239,40 EUR. Alle Preise inkl. 7\% MwSt, zzgl. 44 EUR Versandkosten Inland.

Weitere Informationen und Bestellmöglichkeit direkt beim Springer Kundenservice:

Springer Customer Service Center

Kundenservice Zeitschriften

Haberstr. 7

69126 Heidelberg

Tel. +49/6221/345-4303; Fax: -4229

leserservice@springer.com

www. DerOrthopaede.de 